\title{
A multi-purpose lifting-line flow solver for arbitrary wind energy concepts
}

\author{
Emmanuel Branlard ${ }^{1}$, Ian Brownstein ${ }^{2}$, Benjamin Strom ${ }^{2}$, Jason Jonkman ${ }^{1}$, Scott Dana ${ }^{1}$, and Ian \\ Baring-Gould ${ }^{1}$ \\ ${ }^{1}$ National Renewable Energy Laboratory, Golden, CO 80401, USA \\ ${ }^{2}$ XFlow Energy, Seattle, WA, USA
}

Correspondence: E. Branlard (emmanuel.branlard@nrel.gov)

\begin{abstract}
In this work, we extend the AeroDyn module of OpenFAST to be able to support arbitrary collections of wings, rotors and towers. The new standalone AeroDyn driver supports arbitrary motions of the lifting-surfaces and complex turbulent inflows. We describe the features and updates necessary for the implementation of the new AeroDyn driver. We present different case studies of the driver to illustrate its application to concepts such as: multi-rotors, kites, or vertical axis wind turbines. We perform verification and validation of some of the new features using the following test cases: an elliptical wing, a horizontal axis wind turbine, and a 2D and 3D vertical axis wind turbines. The wind turbine simulations are compared to field measurements. We use this opportunity to point out some limitations of current models and highlight areas which we think should be the focus of future research in wind turbine aerodynamics.
\end{abstract}

\section{Introduction}

Horizontal axis wind turbines (HAWT) have been the main-stream focus of the wind energy community in the past few decades and most aerodynamic tools have been centered around such a concept. This is, for instance, the case for the multi-physics solver OpenFAST (OpenFAST, 2021) developed by the National Renewable Energy Laboratory. The OpenFAST solver has been dedicated to HAWT and cannot ${ }^{1}$ study other wind energy concepts such as: vertical axis wind turbines (VAWT), kites, airborne wind energy concepts, and arbitrary assemblies of rotors and blades/wings. This article attempts to bridge this gap by focusing on adding new aerodynamic functionalities to the aerodynamic model of OpenFAST, named AeroDyn. This first step can later be followed by extending the structural dynamics modules to accommodate these different concepts.

The most common method for the study of a HAWT is the blade element momentum (BEM) method (Glauert, 1935). The method cannot be applied to other concepts, though it inspired the development of streamtube models for vertical axis turbines (De Vries, 1979). General purpose computational fluid dynamics (CFD) solvers are commercially available and have been applied to various wind energy concepts (Makridis and Chick, 2013; Folkersma et al., 2017; Rezaeiha et al., 2017). Their use by the wind energy community is still limited, and dedicated solvers are typically preferred. Such solvers (e.g. Ellipsys (Sørensen, 1995), FLOWer (Weihing et al., 2018), ExaWind (Sprague et al., 2020)) have generic grid-based imple-

\footnotetext{
${ }^{1}$ Airborne wind energy kites have been modeled with the extension to OpenFAST known as KiteFAST (Jonkman, 2021)
} 
mentations, but they have been primarily applied to HAWT. However, simulations of alternative wind energy concepts using these solvers are emerging in the literature (Bangga et al., 2020). CFD applications with arbitrary motions are still challenging and not readily available. Vorticity-based methods have long been considered as the intermediate solution between the computationally intensive CFD methods and the engineering models such as BEM (Perez-Becker et al., 2020; Boorsma et al., 2020). Panel-based methods and lifting-line methods are readily applied to arbitrary assemblies of wings and rotors (Katz and Plotkin, 2001). Generic solvers have been implemented (Grasso et al., 2011; Chatelain et al., 2013; Branlard et al., 2015; Alvarez and Ning, 2019; Boorsma et al., 2020) but often not publicly distributed.

In Section 2, we describe the features of the new AeroDyn driver, the updates to the AeroDyn modules, and briefly mention the implementation. In Section 3, we present different applications of the driver and perform verification and validation of some of its features. We use this opportunity to point out some limitations of current models and highlight areas which we think should be the focus of future research in wind turbine aerodynamics. We conclude our work by summarizing these research questions and providing paths for future work.

\section{Features and implementation}

In this section we describe the main features of the newly-implemented AeroDyn driver. The original AeroDyn driver was limited to the simulation of HAWTs, with a fixed nacelle position, and inflows limited to a power law shear profile (more advanced structural motions and wind conditions can be simulated when coupling AeroDyn within OpenFAST, including aero-elastic effects and turbulence). To be able to model advanced wind energy concepts, the driver was augmented to be able to model rotors and wings of arbitrary geometry, undergoing arbitrary rigid-body motion, and under arbitrary inflows. As such, the driver can be used for configurations that are not currently supported by OpenFAST. We proceed by listing the features of the driver:

- Inflow. The wind field may be defined in three ways: using a uniform power law, a time varying power law (were both the reference velocity and the power coefficient change with time), or using any wind supported by the InflowWind module (OpenFAST, 2021): uniform steady wind, unsteady wind speed and direction (e.g. deterministic gusts), and turbulent wind field of various file formats.

- Geometry. An assembly of fixed or rotating blades/wings is called a "turbine". The driver can have an arbitrary number of turbines. Each turbine comprise of one optional tower and a set of blades. An example of configuration with two turbines is shown in Figure 1. The figure defines the different frames defined for each turbine: the turbine base frame (labeled, t), the nacelle frame (n), the hub frame (h), and the blade frames (b). The labels are used to identify the frame axes and the origins in the following. As indicated in Figure 1, the coordinate systems must be such that the hub rotation occurs about the $x_{h}$ axis, and the blade frame must be such that $x_{b}$ and $y_{b}$ points towards the suction side and the trailing edge respectively when the pitch and twist angles are zero. The turbine base and the tower base have distinct origins but they share the same frame. The tower top is assumed to coincide with the nacelle origin. The origins and orientations of 
each frames are user input, where coordinates are given relative to the parent frame, and orientations are given using the values of three successive rotations (x-y-z Euler angle sequence) taken from the parent frame. A user switch is available to facilitate the input of generic HAWTs geometries. In this framework, an arbitrary wing is setup as a turbine with no rotational speed and an optional tower.
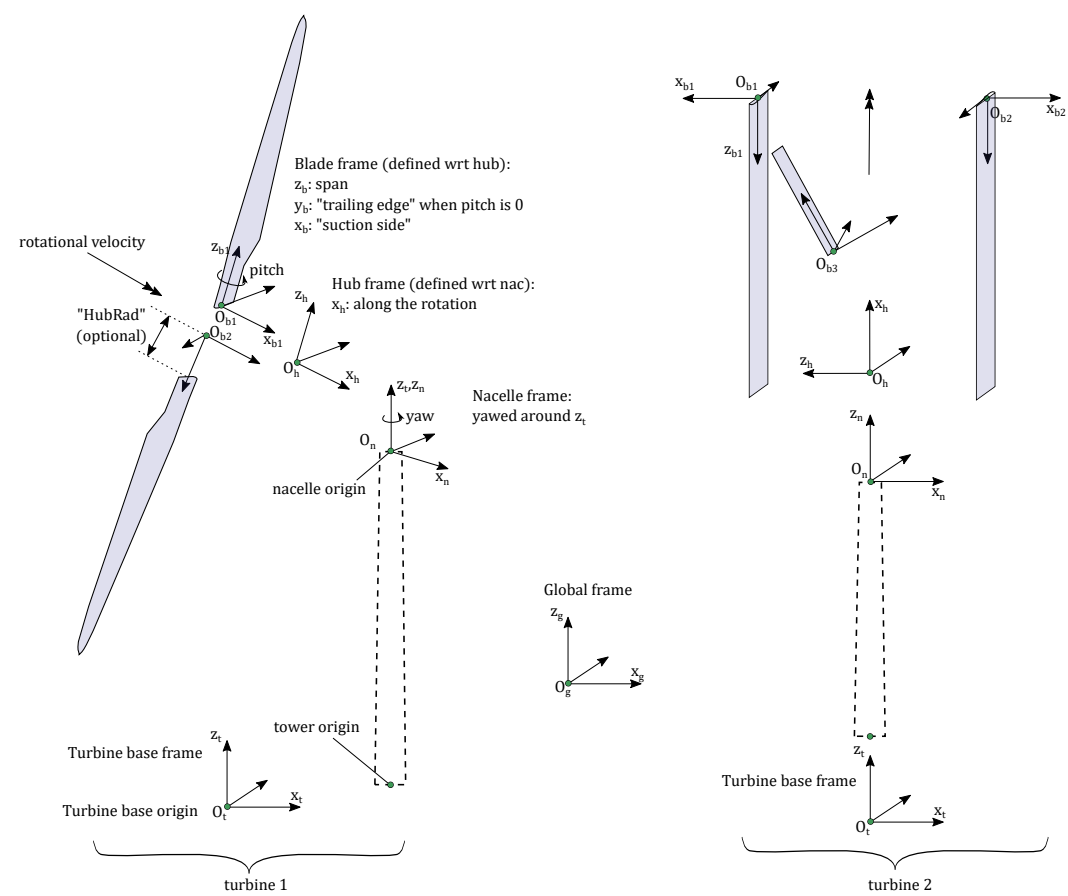

Figure 1. Definition of frames and origins for a two-turbine configuration: a HAWT (left) and a VAWT (right).

- Motion. Motion inputs are provided independently for the base, nacelle, hub, and blades of each turbine. The base motion may be: fixed, sinusoidal in one of six degrees of freedom, or arbitrary. The arbitrary motion is provided using time series of: time, 3 translations, 3 successive rotations, 3 translation velocities, 3 rotational velocities, 3 translational accelerations and 3 rotational accelerations. The nacelle yaws around the $z_{n}$ axis and the user may fix the yaw angle or provide a time series of the nacelle yaw angle, speed and acceleration. The rotor rotates about the $x_{h}$ axis and the user may specify a constant rotational speed or a time varying time series (angular position, speed, and acceleration). Blade pitching occurs around the individual $z_{b}$ axes. The user can specify constant pitch or time series of pitch (position, speed and acceleration) for each individual blade. Non-rotary wings are considered as a special case with 0 rotational speed. The different rigid-body motions are easily implemented using the mesh-mapping routines of OpenFAST called within the AeroDyn driver.

- Flow solver. The driver operates with AeroDyn and the different wake options of AeroDyn can be used to solve the flow. The options currently available are: no induction (using the geometric angle of attack), quasi-steady and dynamic BEM 
for HAWT (Moriarty and Hansen, 2005; Branlard, 2017), or a vortex wake code named OLAF (cOnvecting LAgrangian Filaments) (Shaler et al., 2020). AeroDyn is currently being extended to support hydrokinetic turbines (including buoyancy and added mass effects) and future implementation will include a double-streamtube momentum model for vertical axis turbines. Currently, BEM and OLAF cannot be used simultaneously, but such options will be considered in the future.

- Analysis types. Different analysis types are provided by the driver. In particular, parametric studies can be run by providing a table of combined-case analyses. The reader is referred to the OpenFAST manual for additional details (OpenFAST, 2021).

- Outputs. The driver outputs time series of motion, loads and aerodynamic variables to individual files for each turbine. Additionally, 3D visualization outputs are available for the individual bodies. When OLAF is used, Lagrangian markers and velocity/vorticity planes can be output to visualize the wake.

Changes to the AeroDyn module consisted in supporting multiple rotors throughout the code, with different parameters for each rotor, and extending OLAF so that it can handle an assembly of wings with different number of input sections. In this work, we added two dynamic stall models to AeroDyn: the Boeing-Vertol (BV) model (also present in CACTUS (Murray and Barone, 2011)), and the dynamic stall model of Øye (Øye, 1991; Branlard, 2017). Both models are documented in the OpenFAST documentation (OpenFAST, 2021). The source code of the AeroDyn driver is open-source and available on the OpenFAST repository (OpenFAST, 2021), together with its documentation. Example input files, including some of the cases presented below, are also available and integrated as part of the OpenFAST testing framework.

\section{Applications}

\subsection{Illustrative examples}

We begin the application section by showing visual outputs from simulations done using the AeroDyn driver applied to different wind energy concepts. The vortex wake formulation (OLAF) was used for all simulations because it can be applied to arbitrary geometries and it offers an opportunity to visualize the wake. Visualizations of the wake, blades, towers and velocity planes are given in Figure 2 for: an elliptical wing, a vertical axis wind turbine, a kite performing a "8-figure" and a "quad-rotor" with multiple towers. In the quad rotor figure, the impact of the tower shadow and the wakes is observed in the velocity field. In the remaining of this section, we will dive into specific applications, in order to verify and validate the current implementation. Each investigation will point to research topics for future work on the aerodynamics of wind energy concepts. The points will be summarized in the conclusion. 


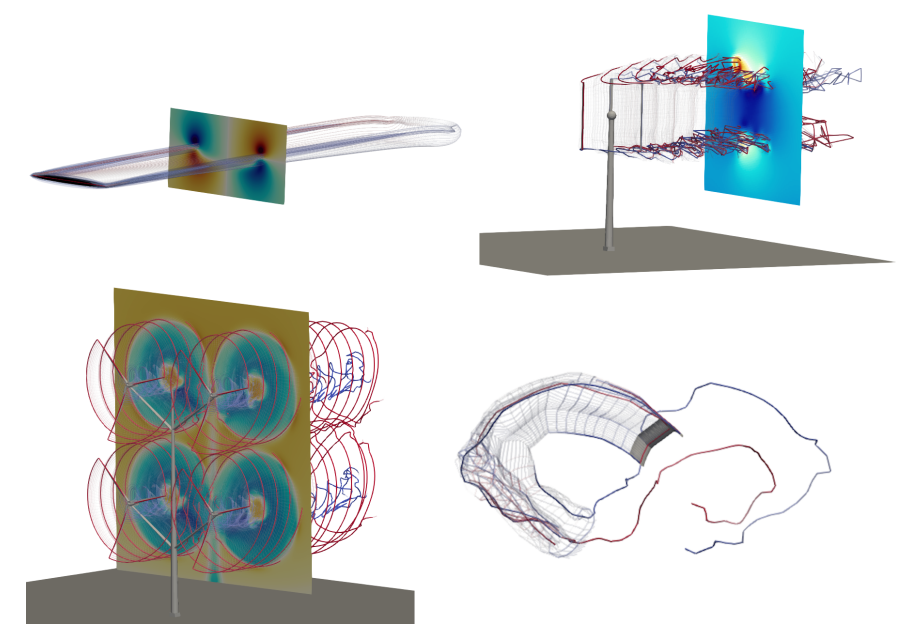

Figure 2. Example of wind energy concepts to which the AeroDyn driver may be applied: elliptical wing, vertical axis turbine, multiple rotors, kites.

\subsection{Elliptical Wing}

In this section we use the elliptical wing test case presented by van Garrel (van Garrel, 2003) to illustrate the capability of the AeroDyn driver in studying isolated lifting lines (not necessarily rotors). The wing span is $b=5 \mathrm{~m}$, the chord $c=c_{0} \sqrt{1-2 y / b}$, with $c_{0}=1 \mathrm{~m}$, the $n+1$ panel nodes are located via a cosine distribution at the spanwise coordinates $y=b / 2 \cos \theta$, with $\theta$ spanning linearly from $-\pi$ to $\pi$. The control points are located between the panel nodes, according to the cosine-approximation algorithm of van Garrel. The wind speed is $1 \mathrm{~m} / \mathrm{s}$ in the chordwise direction and $0.1 \mathrm{~m} / \mathrm{s}$ normal to the chord, leading to a geometrical angle of attack of $5.7106 \mathrm{deg}$. The profile data is uniform along the wing span and set with a linear lift coefficient: $C_{l}(\alpha)=2 \pi \alpha$. The wake convects with the free-stream only (no rollup). Three different number of panels are used for the verification: $n=[20,40,80]$. Baseline results are obtained with no regularization (no "vortex core"), indicated by a zero value of the regularization parameter $\epsilon$. To illustrate the impact of the regularization, simulations for $n=80$ are shown for a regularization parameter proportional to the chord $(\epsilon=0.5 c)$ and a constant parameter $\epsilon=0.1$. The lift coefficient along the span is shown in Figure 3. It was obtained using OLAF, coupled with the AeroDyn driver. The vortex wake results extracted from van Garrel's report are also given in the figure. The strong agreement between the two vortex wake codes supports the verification of OLAF's implementation. Both lifting-line implementations are expected to rely on the same formulation. The results from AeroDyn are reported at the panel nodes and not the control point nodes of OLAF, explaining the minor differences observed towards the wing tips for $n=20$. Under the linear and classical lifting-line approximation of Prandtl (Katz and Plotkin, 2001; Branlard, 2017), the theoretical lift coefficient for the wing is $C_{L, \mathrm{th}}=2 \pi \alpha[1+2 / \mathrm{AR}]^{-1} \approx 0.47653$, where $\mathrm{AR}=b^{2} /\left(\pi b c_{0} / 4\right)$ is the wing aspect ratio. The theoretical value is indicated on the figure. The current simulation setup (cosine distribution without regularization and wake rollup) is well suited to approximate the linear theory, but is not expected 


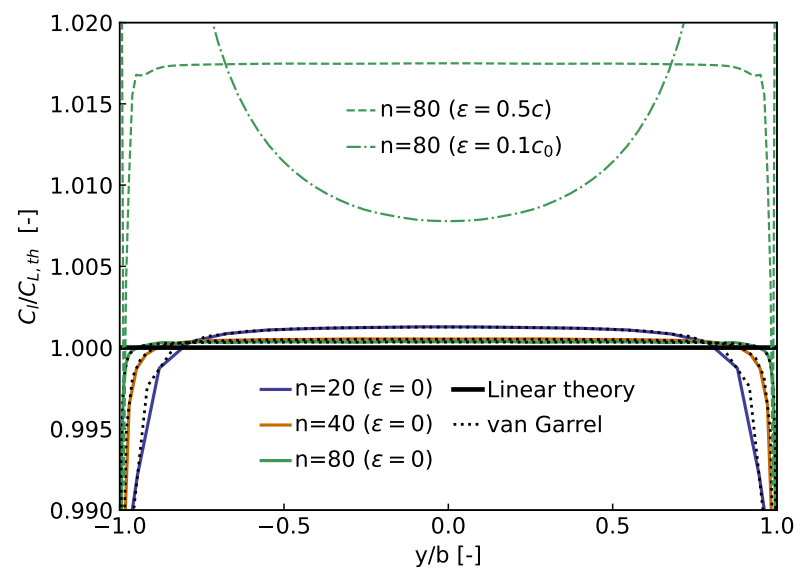

Figure 3. Lift coefficient along elliptical wing $\left(C_{l}\right)$ as predicted by two similar lifting-line implementations (OLAF, and van Garrel) and the linear lifting-line theory $\left(C_{L, t h}\right)$. Results for various number of spanwise stations $(n)$ and regularization parameters $(\epsilon)$.

to match the results fully. To match the linear theory, linear assumptions are needed and the wake needs to follow the chord instead of the freestream. Requirements to match the theory exactly are provided in Chapter 3 of (Branlard, 2017).

A realistic simulation of an elliptical wing requires regularization to account for the physical size of the bound vorticity. This physical size is expected to be related to the size of the boundary layer and the spanwise discretization (Branlard, 2017). The impact of the regularization is clearly observed on Figure 3, and the choice of the regularization parameter can have a drastic impact on the results. The topic of regularization is being actively researched (Martínez-Tossas and Meneveau, 2019; Meyer Forsting et al., 2019; Li et al., 2020).

\subsection{Horizontal axis wind turbine}

AeroDyn was previously dedicated to HAWT and its BEM implementation was extensively tested for such configurations. In this section, we present comparisons between BEM, OLAF and measurements for the 3-bladed NEG-Micon NM80 turbine, rated at $2 \mathrm{MW}$, with a rotor diameter of $80 \mathrm{~m}$. Details about the turbine and the experimental setup is found in the DanAero report (Madsen et al., 2010). We use the test cases from the International Energy Agency (IEA) Wind Task 29 as validation cases (Schepers and et al., 2021). In this work, we present results using the AeroDyn driver for a rigid rotor. Results using OpenFAST for a flexible rotor are given in the IEA Wind Task 29 report, together with a full description of the IEA Wind Task 29 test cases and results from other participants. For the cases presented below, flexibility effects were found to have a negligible impacts on the results.

We begin with case IV.1.2 from the IEA Wind Task 29. The rotor operates at a tip-speed ratio of $\lambda=8.5$ for an average wind speed of $U_{0}=6.1 \mathrm{~m} / \mathrm{s}$. The test case neglects shear and constant uniform inflow is assumed for the simulations. The force coefficients normal and tangential to the chord line are shown in Figure 4. The coefficients were obtained by normalizing the forces with $1 / 2 \pi \rho U_{0}^{2} R$, with $R$ the rotor radius, and $\rho$ the air density. The simulation results shown in Figure 4 are consistent 

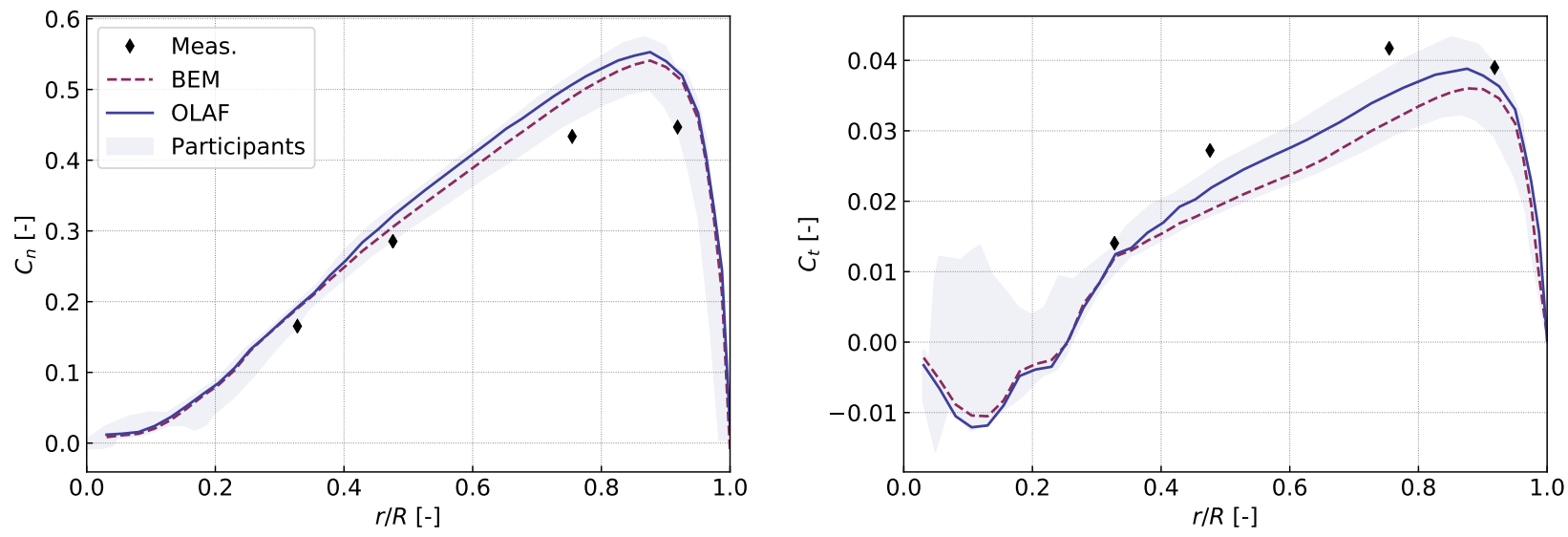

Figure 4. Simulation of a horizontal axis wind turbine using the AeroDyn driver. Results for test case IV.1.2 (constant, uniform inflow) of IEA Task 29. Normal and tangential force coefficients along the blade span (resp. left and right).

with results obtained by other institutions (see Schepers and et al. (2021)), both for the BEM and vortex code. The comparison with measurements is fair but leaves room for improvement. The differences are primarily attributed to the definition of the polar data used by the lifting line codes, which needs to be improved in the follow-up task (Schepers and et al., 2021).

We use cases IV.2.1 and IV.2.2 to study the aerodynamics in sheared and yawed conditions, respectively. Both cases have the same rotational speed and pitch, the tip-speed ratios are 6.9 and 8.0, the yaw angles, 6 and $38^{\circ} \mathrm{deg}$ and the power law exponents, 0.25 and 0.26 respectively. Figure 5 presents the results for both cases as function of the azimuthal position. The normal loads and tangential loads are shown as function of the azimuth, at four radial positions: $r / R=[0.33,0.48,0.75,0.92]$. The azimuth is 0 when the blade is pointing up. Both elastic (with ElastoDyn) and rigid (with AeroDyn driver) simulations were performed. Some differences are observed between the two (comparing dashed and plain lines of the same color) but these differences are not as pronounced as the differences between the BEM and OLAF (comparing blue and red curves). The vortex code agrees significantly better with the measurements than the BEM method for the yawed case. The shear-only case appears to be challenging, especially towards the root. The reasons for such discrepancies will require further investigation.

\subsection{Vertical axis turbine}

\subsubsection{D case}

In this section, we use the 2D vertical axis wind turbine (VAWT) model presented by Ferreira et al.(Ferreira et al., 2014): a two-bladed turbine of radius $R=1 \mathrm{~m}$, with blades of constant chord $c=0.1 \mathrm{~m}$, and $15 \%$ relative thickness. The lift coefficient is set to $C_{l}=2 \pi 1.11 \sin \alpha$ and the drag and moment coefficients are zero. The tip-speed ratio is $\lambda=4.5$. Simulations were run using the vortex code CACTUS (Murray and Barone, 2011) and with OLAF, and compared with double multiple stream tube model (DMST) from (Ferreira et al., 2014). The angle of attack as function of azimuth is shown in Figure 6. The vortex codes CACTUS and OLAF are observed to strongly agree in this case for the estimation of the angle of attack. CACTUS uses a vortex 

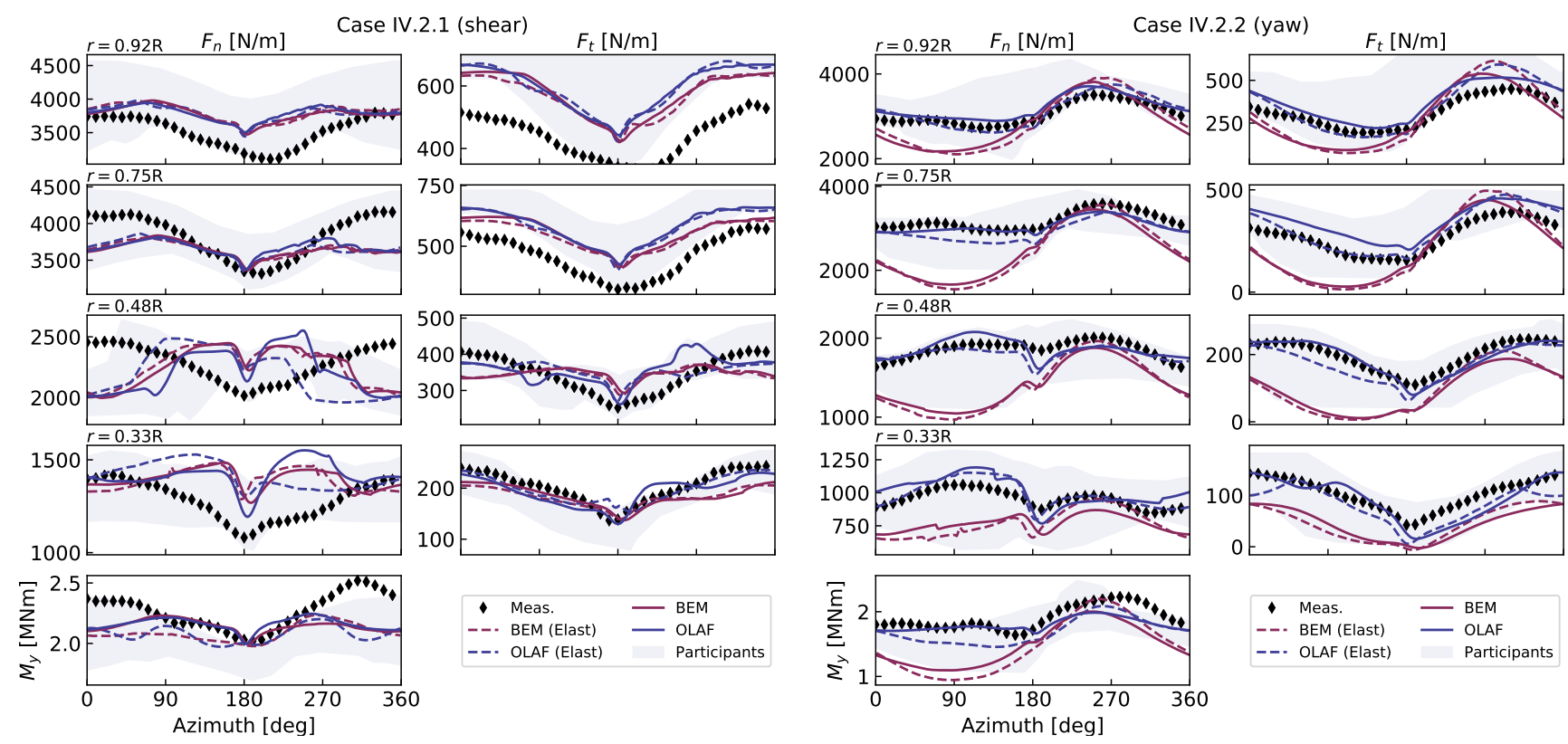

Figure 5. Results for a horizontal axis wind turbine (NM 80) under strong shear (left) and yawed (right) conditions. The normal $\left(F_{n}\right)$ and tangential $\left(F_{t}\right)$ loads are shown at four radial positions as function of the azimuth. The blade root flapping moment $M_{y}$ is shown at the bottom. Elastic ("Elast") and rigid simulations are compared to the DanAero measurements.

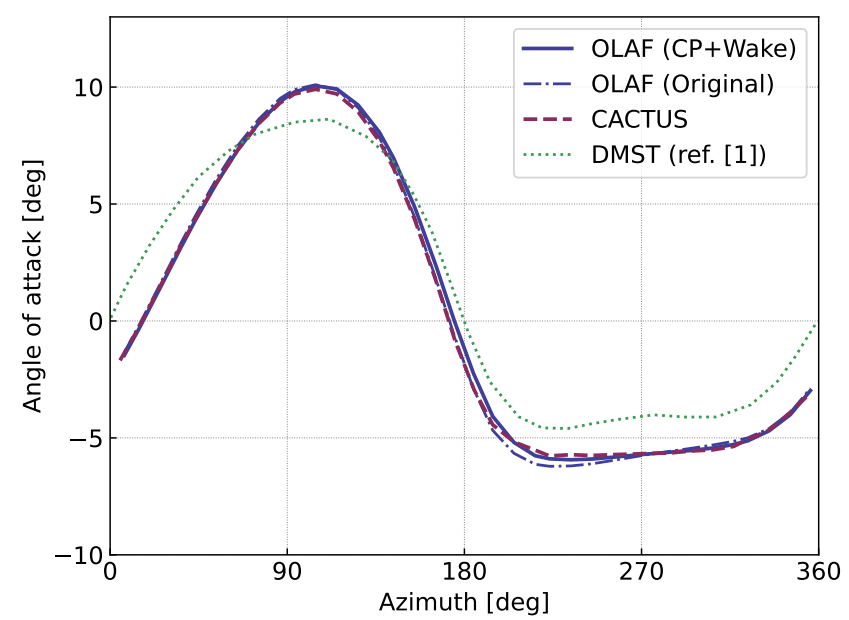

Figure 6. Angle of attack on a 2D VAWT as obtained with various vortex methods and with the double multiple stream tube theory.

formulation where the velocity at control points is obtained from the average of the velocity at the nodes, and where the wake is being shed at the lifting-line. The original OLAF formulation uses the induced velocity obtained in between nodes and sheds the wake at the trailing edge of the blade. For this work, OLAF was modified so as to be able to have a similar formulation 

power coefficient is shown in Figure 7 for a simulation at $\lambda=4.5$. From the figure, it is observed that the choice of dynamic

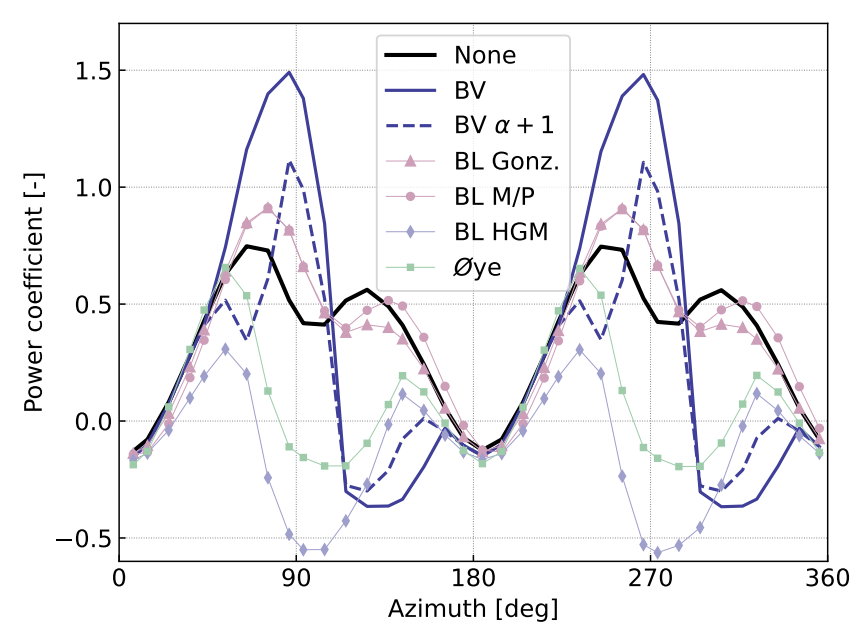

Figure 7. Influence of the choice of dynamic stall model on the power coefficient of a $2 \mathrm{D}$ vertical axis wind turbine

as CACTUS. In the case presented in Figure 6, it is seen that by using the same formulation (i.e. comparing CACTUS and $\mathrm{OLAF}$ " $\mathrm{CP}+$ Wake" on the figure) a slightly better agreement is obtained. A more significant impact of the implementation was observed on other simulations. The choice of implementation of lifting-line vortex methods (shedding at trailing edge, location of control points) remains an open question.

The previous test case doesn't activate the dynamic stall model ${ }^{2}$ as a results of the low angle of attack and artificial liftcoefficient used. We replaced the polar data with a realistic polar data of a NACA0015 airfoil which stalls at approximately 8.5 deg. The angle of attack is similar to the one obtained in Figure 6, oscillating between $\pm 10 \mathrm{deg}$, but the dynamic stall has a strong influence on the lift coefficient and power coefficient. In this work, we implemented the Boeing-Vertol (BV) model, and the dynamic stall model of Øye. AeroDyn also includes three variations of the Beddoes-Lesihman (BL) model (Leishman and Beddoes, 1989): the Gonzalez's (BL Gonz.) and the Minemma/Pierce (BL MP) variants (Damiani and Hayman, 2019), and the 4-states model from Hansen et al. (Hansen et al., 2004) (BL HGM). The impact of the choice of the dynamic stall on the power coefficient is shown in

stall model has a dramatic impact on the aerodynamic performances. It is common practice in the VAWT community to tune the parameters of the dynamic stall model such as to achieve performances that matches the measurements. To illustrate this, we increased the stall angle parameter of the Boeing-Vertol model by 1 deg (labeled as "BV $\alpha+1$ " on the figure). Again, such a change has a strong impact on the response, delaying the onset and activation of the dynamic model. It is clear how such tuning of the coefficients can lead to desired responses and performances. Overall, the spread of results indicates that dynamic stall models for VAWT (and likely HAWT) should be the topic of future research.

\footnotetext{
${ }^{2}$ In this article, we use the term "dynamic stall" to refer to unsteady aerodynamics effects on an airfoil section (including unsteady attached flows).
} 


\subsubsection{D case, comparison with measurements}

In this section, we model a prototype 5-kW VAWT with the new AeroDyn driver. The turbine consists of 9 blades: 3 vertical blades, each attached to the hub by two support arms. A picture of the wind turbine is shown in Figure 8. The turbine was designed and constructed by XFlow Energy and was tested at the Field Laboratory for Optimized Wind Energy (FLOWE) in Lancaster, CA. The turbine was tested between February and April 2020. The field measurements were collected using two

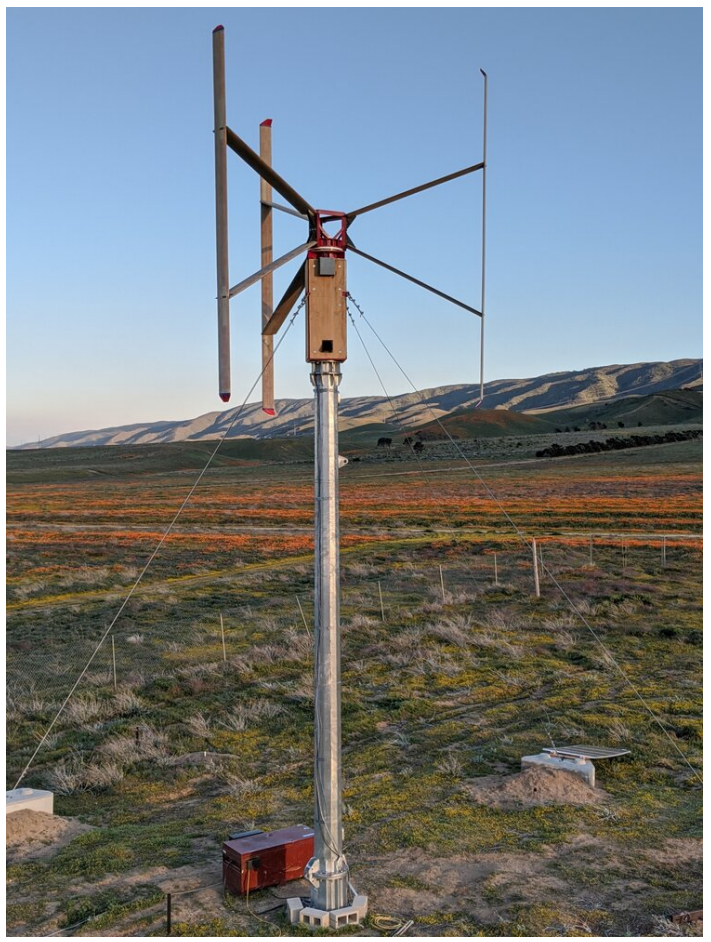

Figure 8. Photo of XFlow's 5-kW prototype VAWT at the Field Laboratory for Optimized Wind Energy in Lancaster, CA

6-axis load cells mounted between the vertical blades and its support arms. The load cells were custom units developed by Sensing Systems from Dartmouth, MA. The wind speed was measured using a pair of APRS \#40R anemometers positioned 2 rotor diameters upstream of the rotor. The measurements presented have had inertial effects subtracted.

First, we run simulations with steady inflow and constant rotational speed to evaluated the power curve of the turbine. The power coefficient as function of tip-speed ratio are compared to field measurements in Figure 9. For both vortex codes, the power coefficient was corrected to account for excrescences as follows:

$C_{P}=C_{P, \text { clean }}-\Omega \frac{\left[C_{Q, \text { exc }} 1 / 2 \rho\left(R^{2}\right) R(\Omega R)^{2}\right]}{1 / 2 \rho\left(2 R^{2}\right) U_{0}^{3}}$

where $C_{P, \text { clean }}$ is the power coefficient obtained from the vortex code and the term in bracket is the excrescences torque. The excrescences torque coefficient was evaluated experimentally to $C_{Q \text {,exc. }}=0.009$. In Figure 9, the results labeled "OLAF 


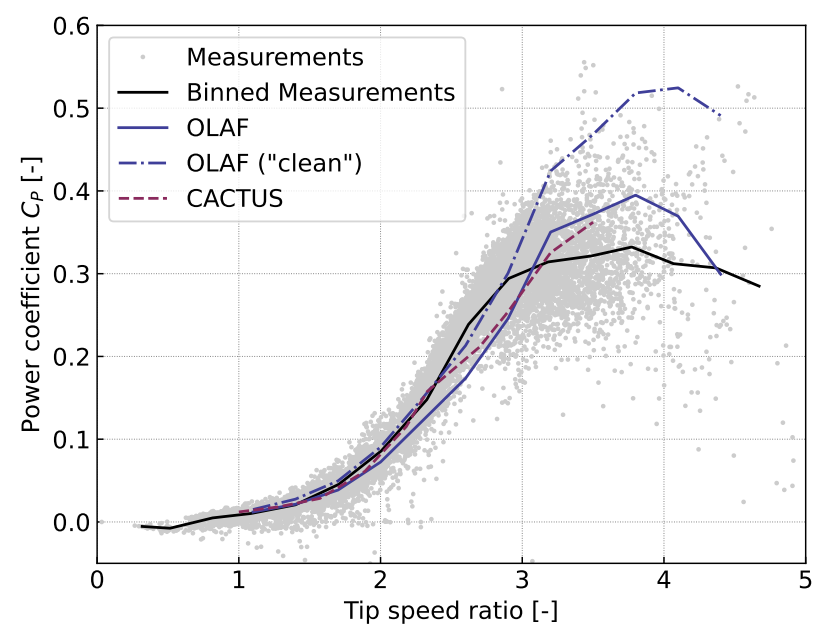

Figure 9. Performance of the VAWT model as obtained with the simulation tools OLAF and CACTUS and compared with measurements. The curve "OLAF (clean)" does not include excrescences.

(clean)" are results without the excrescences, whereas the label "OLAF" and "CACTUS" include the excrescences. We observed in Figure 9 that both vortex codes capture the main characteristics of the power curve. Despite a similar implementation used between OLAF and CACTUS, some differences of outputs for this advanced structure are observed. The performances obtained using OLAF appear to be under predicted below $\lambda=3$ and over predicting otherwise. It is noted that the dynamic stall coefficients of the Boeing-Vertol model were tuned such that the CACTUS simulation would match the measured power curve. It is expected that another tuning of the dynamic stall coefficients for OLAF would lead to stronger agreement with the measurements.

We illustrate the differences between the models by looking at time traces of the total force on the first vertical blade at different tip-speed ratios. Dimensionless force coefficients are computed as $C=F /\left(1 / 2 \rho(2 R)^{2} U_{0}^{2}\right)$, where $F$ is the force in a given direction. The forces are reported in the coordinate of the blade as illustrated in Figure 1. The force coefficients obtained from field measurements and simulation are compared in Figure 10. To demonstrate the capabilities of the AeroDyn driver, simulations with shear and turbulence were also carried on. The power law profiles and turbulence intensities from the field measurements were used to generate synthetic turbulent inflow with TurbSim (Jonkman and Buhl, 2006). Results from these simulations, averaged over 24 revolutions, are indicated by the label "OLAF (turb)" on figure Figure 10. The azimuthal positions $90^{\circ}$ and $270^{\circ}$ correspond to the position where the blade is upwind and downwind respectively. A fair agreement with the measurements is obtained for both tools. The response when the blade is in the wake $\left(270^{\circ}\right)$ appear more challenging to capture, in particular at higher tip-speed ratios and for the tangential coefficient $\left(C_{y}\right)$. This likely indicate issues related to the estimation of the drag force or the account of viscous effects in the wake. In general, a strong agreement is observed between OLAF and CACTUS. Spikes observed in the CACTUS simulations are not present in the OLAF runs, which displays 

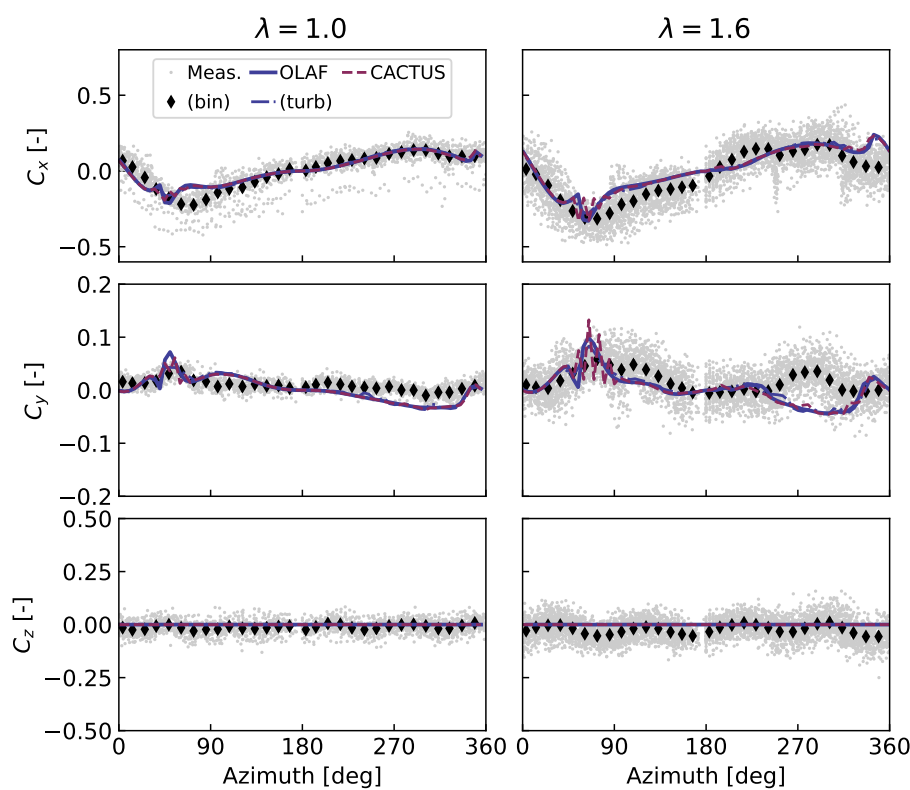
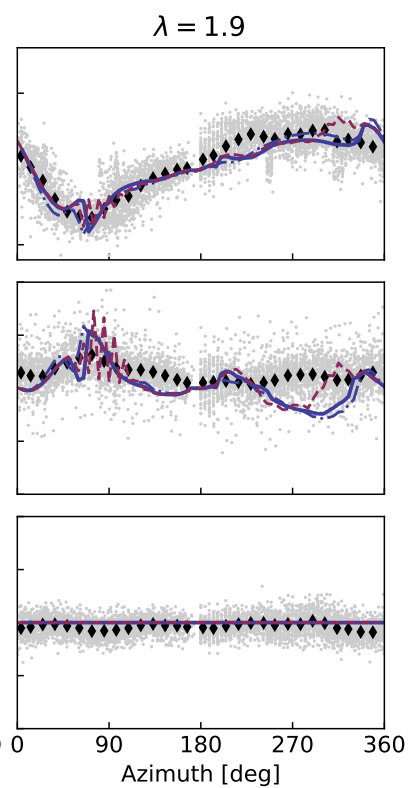
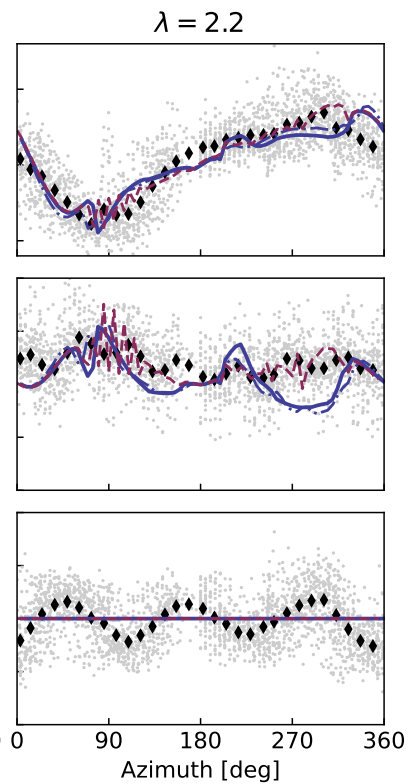

Figure 10. Force coefficients as measured and simulated on the vertical axis turbine model.

a smoother response. The differences between the turbulent and uniform simulations appear to be minor for these cases but are expected to become more important for larger shear and turbulence intensities.

Based on finite element analysis of XFlow's 5-kW turbine geometry, we expect $C_{x}$ to be the least affected by aero-elastic effects. This agrees well with the simulation, and is a possible explanation for discrepancies between $C_{y}$ and $C_{z}$ from the field and simulation. Based on the finite element analyses, the turbine's first mode of excitation corresponds to a vertical motion of the blades, which is observed to be a dominant effect in the field measurements. Because of this, it is not surprising that the rigid-body AeroDyn/OLAF simulations did not capture the oscillations observed in $C_{z}$. Future work coupling OLAF with an elastic solver should more accurately capture this effect.

\section{Conclusions}

In this work, we described the features of a general-purpose driver to perform aerodynamic simulations of wind energy concepts. Different applications were presented to highlight the versatility of the driver and point to areas of research, namely:

- The regularization parameter of lifting-line vortex methods, commonly referred to as the "vortex core" has a strong impact on the accuracy of the results, and should be further investigated.

- Different lifting-line vortex code implementations can lead to different loads and induction field, depending on the choice of formulation. 
https://doi.org/10.5194/wes-2021-99

Preprint. Discussion started: 14 September 2021

(c) Author(s) 2021. CC BY 4.0 License.

(c) (i)

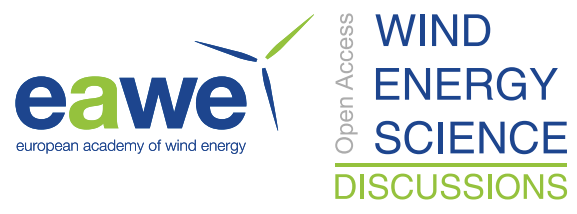

- The blade element momentum theory is challenged by out-of-plane situations (yaw, shear and coning), and, despite the ad-hoc corrections available, the method does not capture all the trends observed in measurements.

- The choice of dynamic stall model significantly impacts the simulation results of VAWT, and practitioners commonly fall back to tuning the parameters of the model, in lack of a universal and reliable model.

Aerodynamic concepts different from the widely studied horizontal axis turbines, offer a variety of aerodynamic challenges. The new aerodynamic driver opens the door for further investigation of these concepts. Targeted aerodynamic studies within a controlled environment can be carried on using the new prescribed motion feature. The feature is relevant for future aerodynamic research areas, including: floating offshore wind turbines or unsteady aerodynamics effects under (prescribed) elastic motions (e.g. flutter). The aerodynamic models currently implemented in AeroDyn consists of the Blade Element Momentum method (both quasi-steady and dynamic) and a lifting-line vortex lattice solver. AeroDyn will soon be extended to support hydro-kinetic turbines. Additional models will also be added in the future, such as the double multiple streamtube model, and mixed formulations between BEM and vortex methods. 
https://doi.org/10.5194/wes-2021-99

Preprint. Discussion started: 14 September 2021

(C) Author(s) 2021. CC BY 4.0 License.

\section{References}

Alvarez, E. J. and Ning, A.: Modeling Multirotor Aerodynamic Interactions Through the Vortex Particle Method, in: AIAA Aviation Forum, Dallas, TX, https://doi.org/10.2514/6.2019-2827, 2019.

Bangga, G., Dessoky, A., Wu, Z., Rogowski, K., and Hansen, M. O.: Accuracy and consistency of CFD and engineering models for simulating vertical axis wind turbine loads, Energy, 206, https://doi.org/10.1016/j.energy.2020.118, https://ideas.repec.org/a/eee/energy/ v206y2020ics0360544220311944.html, 2020.

Boorsma, K., Wenz, F., Lindenburg, K., Aman, M., and Kloosterman, M.: Validation and accommodation of vortex wake codes for wind turbine design load calculations, Wind Energy Science, 5, 699-719, 2020.

Branlard, E.: Wind Turbine Aerodynamics and Vorticity-Based Methods: Fundamentals and Recent Applications, Springer International Publishing, https://doi.org/10.1007/978-3-319-55164-7, 2017.

Branlard, E., Papadakis, G., Gaunaa, M., Winckelmans, G., and Larsen, T. J.: Aeroelastic large eddy simulations using vortex methods: unfrozen turbulent and sheared inflow, Journal of Physics: Conference Series (Online), 625, https://doi.org/10.1088/17426596/625/1/012019, 2015.

Chatelain, P., Backaert, S., Winckelmans, G., and Kern, S.: Large Eddy Simulation of Wind Turbine Wakes, Flow Turbulence And Combustion, 91, 587-605, https://doi.org/10.1007/s10494-013-9474-8, 2013.

Damiani, R. and Hayman, G.: The Unsteady Aerodynamics Module for FAST 8, Tech. rep., National Renewable Energy Laboratory, nREL/TP-5000-66347, 2019.

De Vries, O.: Fluid dynamic aspects of wind energy conversion, AGARD report, Brussels, Belgium, AG-243, 1-50, 1979.

Ferreira, C. S., Madsen, H. A., Barone, M., Roscher, B., Deglaire, P., and Arduin, I.: Comparison of aerodynamic models for Vertical Axis Wind Turbines, Journal of Physics: Conference Series, 524, https://doi.org/10.1088/1742-6596/524/1/012125, 2014.

Folkersma, M., Schmehl, R., and Viré, A.: Fluid-Structure Interaction Simulations on Kites, in: Airborne Wind Energy Conference 2017, AWEC 2017 ; Conference date: 05-10-2017 Through 06-10-2017, pp. 144-144, https://doi.org/10.4233/uuid:4c361ef1-d2d2-4d14-9868$16541 \mathrm{f} 60 \mathrm{edc} 7,2017$.

Glauert, H.: Airplane propellers, Division L, in: Aerodynamic Theory, Volume IV, edited by W.F. Durand (ed), Julius Springer, Berlin, 1935.

Grasso, F., van Garrel, A., and Schepers, G.: Development and Validation of Generalized Lifting Line Based Code for Wind Turbine Aerodynamics, Tech. Rep. ECN-M-11-004, ECN, 2011.

Hansen, M., Gaunaa, M., and Aagaard Madsen, H.: A Beddoes-Leishman type dynamic stall model in state-space and indicial formulations, Tech. rep., Risø National Laboratory, Roskilde, Denmark, 2004.

Jonkman, B. and Buhl, M.: TurbSim User's Guide, Tech. rep., National Renewable Energy Laboratory, nREL/TP-500-39797, 2006.

Jonkman, J.: Makani Energy Kite Modeling - Cooperative Research and Development Final Report, Tech. Rep. NREL/TP-5000-80635, National Renewable Energy Laboratory, 2021.

Katz, J. and Plotkin, A.: Low-Speed Aerodynamics, 2nd Edition, Cambridge Aerospace Series(No. 13), Cambridge University Press, 2001. Leishman, J. G. and Beddoes, T.: A semi-empirical model for dynamic stall, Journal of the American Helicopter Society, 34, p3-17, 1989. Li, A., Gaunaa, M., Pirrung, G. R., Ramos-García, N., and Horcas, S. G.: The influence of the bound vortex on the aerodynamics of curved wind turbine blades, Journal of Physics: Conference Series, 1618, 052 038, https://doi.org/10.1088/1742-6596/1618/5/052038, https://doi.org/10.1088/1742-6596/1618/5/052038, 2020.

Madsen et al.: The DAN-AERO MW Experiments - Final Report, Tech. Rep. Riso-R-1726, Ris $\varnothing-D T U, 2010$. 
https://doi.org/10.5194/wes-2021-99

Preprint. Discussion started: 14 September 2021

(C) Author(s) 2021. CC BY 4.0 License.

Makridis, A. and Chick, J.: Validation of a CFD model of wind turbine wakes with terrain effects, Journal of Wind Engineering and Industrial Aerodynamics, 123, 12-29, https://doi.org/https://doi.org/10.1016/j.jweia.2013.08.009, https://www.sciencedirect.com/science/article/pii/ S0167610513001827, 2013.

Martínez-Tossas, L. A. and Meneveau, C.: Filtered lifting line theory and application to the actuator line model, Journal of Fluid Mechanics, 863, 269-292, https://doi.org/10.1017/jfm.2018.994, 2019.

Meyer Forsting, A. R., Pirrung, G. R., and Ramos-García, N.: A vortex-based tip/smearing correction for the actuator line, Wind Energy Science, 4, 369-383, https://doi.org/10.5194/wes-4-369-2019, https://wes.copernicus.org/articles/4/369/2019/, 2019.

Moriarty, P. J. and Hansen, A. C.: AeroDyn Theory Manual, Tech. rep., National Renewable Energy Laboratory, nREL/EL-500-36881, 2005.

Murray, J. and Barone, M.: The development of CACTUS : a wind and marine turbine performance simulation code., in: 49th AIAA Aerospace Sciences Meeting, Orlando, Florida, 2011.

OpenFAST: Open-source wind turbine simulation tool, available at http://github.com/OpenFAST/OpenFAST/, 2021.

Øye, S.: Dynamic stall, simulated as a time lag of separation, Proceedings of the 4th IEA Symposium on the Aerodynamics of Wind Turbines, 1991.

Perez-Becker, S., Papi, F., Saverin, J., Marten, D., Bianchini, A., and Paschereit, C. O.: Is the Blade Element Momentum theory overestimating wind turbine loads? - An aeroelastic comparison between OpenFAST's AeroDyn and QBlade's Lifting-Line Free Vortex Wake method, Wind Energy Science, 5, 721-743, https://doi.org/10.5194/wes-5-721-2020, 2020.

Rezaeiha, A., Kalkman, I., and Blocken, B.: CFD simulation of a vertical axis wind turbine operating at a moderate tip speed ratio: Guidelines for minimum domain size and azimuthal increment, Renewable Energy, 107, 373-385, https://doi.org/https://doi.org/10.1016/j.renene.2017.02.006, https://www.sciencedirect.com/science/article/pii/S0960148117300848, 2017.

Schepers, J. G. and et al.: Final report of Task 29, Phase IV: Detailed Aerodynamics of Wind Turbines, Tech. rep., IEA Wind, Task $29,2021$. Shaler, K., Branlard, E., and Platt, A.: OLAF User's Guide and Theory Manual, Tech. rep., National Renewable Energy Laboratory, nREL/RP-5000-75959, 2020.

Sørensen, N. N.: General purpose flow solver applied to flow over hills, Ph.D. thesis, Risø-DTU, 1995.

Sprague, M., Ananthan, S., Vijayakumar, G., and Robinson, M.: ExaWind: A multifidelity modeling and simulation environment for wind energy, Journal of Physics: Conference Series, 1452, 012 071, https://doi.org/10.1088/1742-6596/1452/1/012071, 2020.

van Garrel, A.: Development of a wind turbine aerodynamics simulation module, Tech. Rep. ECN-C-03-079, ECN, 2003.

Weihing, P., Letzgus, J., Bangga, G., Lutz, T., and Krämer, E.: Hybrid RANS/LES Capabilities of the Flow Solver FLOWer-Application to Flow Around Wind Turbines, in: Progress in Hybrid RANS-LES Modelling, edited by Hoarau, Y., Peng, S.-H., Schwamborn, D., and Revell, A., pp. 369-380, Springer International Publishing, Cham, 2018. 